\title{
The Potential of Topographical Feedforward Neural Network (T-FFNN) Technique in Monthly Wind Speed and Direction Prediction
}

\author{
S. M. Lawan \\ Department of Electrical \\ Engineering \\ Faculty of Engineering, Kano \\ University of Science and \\ Technology, Wudil \\ Kano State, Nigeria \\ salisumuhdlawan@gmail.com
}

\author{
W. A. W. Z. Abidin \\ Department of Electrical and \\ Electronic Engineering \\ Faculty of Engineering, \\ Universiti Malaysia Sarawak \\ 94300, Kota Samarahan, Sarawak \\ Malaysia \\ wzaazlan@unimas.my \\ I. Abba \\ Department of Electrical \\ Engineering \\ School of Technology, Kano \\ State Polytechnic \\ Kano State \\ ibrahimaba12950@yahoo.com
}

\author{
A. M. Lawan \\ Department of Mathematical \\ Science \\ Faculty of Science, Bayero \\ University Kano \\ Kano State Nigeria \\ aminalawan25@yahoo.com
}

\author{
S. L. Bichi \\ Department of Mathematical \\ Science \\ Faculty of Science, Bayero \\ University Kano \\ Kano State Nigeria \\ slawanbh@gmail.com
}

\begin{abstract}
In this paper, the time series, and a parametric feedforward neural network model were designed. A methodology for wind speed prediction in the regions where wind speed is not available by measurement based on the T-FFNN is proposed in this work according to the meteorological, topographical and geographical parameters for long-term prediction. Typical wind speed and direction are respectively predicted by the optimum 9-152-1 T-FFNN. Then the prediction results are analyzed. The results show that the suggested approach is powerful and can be used effectively to predict the wind speed and direction. The observed and modeled data were used in developing the energy map using ArcGIS 9.3 which shows the distribution of wind speed and power density across the studied area.
\end{abstract}

Keywords-wind, prediction, wind mapping, neural network,

\section{INTRODUCTION}

Wind speed and direction are the most important parameters in studying the potential of wind energy and micrositing analysis. In fact, it is well-know that the wind power varies directly as cubic of wind speed. Many experts have claimed that an error of $1 \%$ in wind speed measurement cause of around $2 \%$ deviation in energy production [1]. For that reason, it is of utmost importance to predict wind speed with acceptable accuracy.

Prior to the soft computing, wind speed and direction predictions were normally carried out using physical equations. For instance, mass consistent-code, multi-grid, continuity equation, boundary layer flow and so on [2]. These approaches tend to be complex due to irregularities and unpredictable nature of wind flows. Furthermore, no mathematical model either physical or numeric provides a perfect, definitive solution [3]. As such soft computing that involves fuzzy logic and neural networks (NN) are discovered to be more acceptable [4]. Simulating annealing (SA), support vector machine (SVM) and fuzzy logics are usually applied to the prediction of short samples. Moreover, according to [5] in dealing with nonlinear system modeling, these methods have some drawbacks such as difficulty in selecting the parameters and complexity in computation time.

The ANN can be applied conveniently without the need of mathematical formulation, or in which a numerical model can hardly be constructed. In recent times, ANN has been employed for wind speed and power predictions, because of the fault tolerance, simple topology, fast computing time, parallelism and ability to estimate nonlinear functions [4, 6-10] Most of the reported studies have considered different meteorological parameters in developing wind speed prediction models. However, none of these studies have included the effects of roughness length and terrain elevation. Based on these ANN advantages and the effects of terrain in wind flow, a T-FFNN is proposed.

\section{Methodology}

\section{A. Study Area Description}

Sarawak is one of the two Malaysia states located on the island of Borneo. The administrative capital is situated in Kuching. The state population was 2, 3999,893. Due to its large area, it has the lowest population density in Malaysia, standing at 22 people per $\mathrm{km}^{2}$. The state is located between 